\title{
INTERESSE, EXPERIÊNCIA, AUTOEFICÁCIA, AUTORREGULAÇÃO E CARGA COGNITIVA: IMPLICAÇÕES PARA A APRENDIZAGEM SIGNIFICATIVA DA FÍSICA*
}

Interest, experience, self-efficacy, self-regulation, and cognitive load: implications for meaningful learning of physics

\author{
Marco Antonio Moreira ${ }^{1}$ \\ ${ }^{1}$ Instituto de Física - UFRGS
}

\begin{abstract}
Resumo
Este texto é a versão artigo de uma apresentação feita, em diapositivos, em dois congressos de Ensino de Física. Sua proposta é a de chamar atenção de professores (as) sobre a relevância de determinadas variáveis afetivas e cognitivas no processo ensino-aprendizagem da Física, na Educação Básica e Superior, independente de estratégias instrucionais. São destacadas variáveis como interesse, autoeficácia e carga cognitiva, entre outras.
\end{abstract}

Palavras-chave: Ensino de Física. Aprendizagem Significativa. Variáveis afetivas e cognitivas.

\footnotetext{
*Confêrencia plenária na XIV Conferencia Interamericana sobre Enseñanza de la Física, virtual, UNED, Costa Rica, 5 a 9 de julho de 2021; Conferência de abertura do XXIV Simpósio Nacional de Ensino de Física, virtual, UFABC, Brasil, 19 a 23 de Julho de 2021.
} 


\begin{abstract}
This text in the article version of a slide presentation in two Physics Teaching Meetings. Its proposal is to call the teacher's attention to the relevance of some affective and cognitive variables in the teaching-learning process of physics, both in basic and higher education, independently of instructional strategies. Variables such as interest, self-efficacy, and cognitive load, among others, are emphasized.
\end{abstract}

Keywords: Physics teaching. Meaningful Learning. Affective and cognitive variables.

\title{
I. INTRODUÇÃO
}

Conceitos-chave das teorias de John Dewey (interesse e experiência), Albert Bandura (autoeficácia e autorregulação) e John Sweller (carga cognitiva intrínseca e extrínseca) serão destacados como essenciais para a aprendizagem significativa de conhecimentos físicos. Interesse não é somente motivação, experiência não é simplesmente atividade experimental. Crenças de autoeficácia e autorregulação têm grande influência no comportamento humano, inclusive na aprendizagem, ou não, de novos conhecimentos. Carga cognitiva é muito mais do que quantidade de problemas; um único problema pode implicar alta carga cognitiva, mas pode ser reduzida dependendo de como é proposto. De um modo geral, o ensino da Física não desperta o interesse dos alunos, gera crenças de baixa autoeficácia e não leva em consideração o papel das memórias de curto e longo prazo na aprendizagem. Este panorama tem que mudar se o que se espera é aprendizagem significativa da Física.

\section{A teOriA dA EXPERIÊNCIA E INTERESSE DE JOHN DEWEY}

Que educação não é uma questão de narrar e ouvir, mas sim um processo ativo e construtivo, é um princípio geralmente violado na prática (Dewey, 2018, p.43;1916).

Se todos os envolvidos na educação se dessem conta que a qualidade do processo mental , não a produção de respostas corretas, é a medida do crescimento educacional, algo como uma revolução no ensino aconteceria (op.cit, p. 187).

São falas de Dewey há mais de 100 anos (a primeira edição de sua obra Educação para a Democracia é de 1916), mas a cultura da resposta correta, o treinamento para as provas (teaching for testing) continua vigente. Escolas e professores têm que se submeter a essa "educação".

\section{II.1. Experiências}

Dewey defendia a importância das experiências como estratégia de ensino e criticava a educação tradicional por não dar atenção às experiências de aprendizagem dos alunos.

No ensino, aprendizes deveriam ter experiências de qualidade que, junto com suas experiências já existentes, levassem à aprendizagem. 
Mas, para Dewey experiências não são meras atividades. Não estão associadas principalmente ao conhecimento, mas às vidas e ao modo de viver, abrangendo relações entre o sujeito e o mundo.

\section{INTERESSE}

A suposição de que a aprendizagem ocorre através de experiências não significa que quaisquer experiências sejam educativas. Tudo depende da qualidade da experiência e essa qualidade tem dois aspectos: o imediato refere-se a ser agradável ou desagradável e o outro tem a ver com sua influência em experiências posteriores, i.e., desejáveis experiências futuras (Dewey, 2015). Isso leva ao conceito de interesse.

Despertar o interesse dos alunos pelos conteúdos, declarativos ou procedimentais, que estão sendo trabalhados nas aulas, assim como por experiências de qualidade, é um grande desafio para o ensino. Provavelmente, o maior de todos.

Segundo Dewey (1916) a palavra interesse sugere, etimologicamente, o que está entre, aquilo que conecta duas coisas que estariam distantes se não existisse.

$\mathrm{Na}$ aprendizagem, as capacidades do aluno constituem o estágio inicial; os objetivos do professor representam um limite remoto. Entre os dois está o meio, ou seja, condições intermediárias; ações a serem cumpridas; dificuldades a serem superadas; aplicações para serem feitas. Somente através delas, em certo tempo, é que atividades iniciais alcançarão uma consumação satisfatória.

Interesse significa que quem está aprendendo se identifica com os objetos de estudo que definem a atividade e fornecem meios e obstáculos a sua realização (op cit.).

\section{CARACTERÍsticas DO INTERESSE}

Recentemente, Renninger, Nieswandt e Hidi (2015) identificaram cinco características do interesse que são consensuais entre todos os que o estudam como uma variável pedagógica distinta:

- interesse sempre refere-se à interação com algum conteúdo particular (e.g., matemática, ciências, letras);

- interesse existe em uma relação particular entre o aprendiz e seu entorno;

- interesse tem tanto componentes afetivos como cognitivos; a influência de cada um varia dependendo do estágio de desenvolvimento do interesse;

- o aprendiz pode, ou não, estar consciente de que seu interesse foi despertado;

- interesse funciona como uma recompensa que leva o aprendiz a procurar novos recursos e desafios. 


\section{FASES DE DESENVOLVIMENTO DO INTERESSE}

Quatro fases caracterizam o desenvolvimento do interesse (op.cit.,p.4):

1. situacional despertadora;

2. situacional mantida;

3. situacional emergente;

4. interesse individual bem desenvolvido.

Nas fases iniciais (interesse despertado e mantido) aprendizes precisam de apoio para fazer conexões entre tarefas e o mundo real.

Nas fases posteriores (interesse emergente e individual bem desenvolvido) essas conexões estão feitas e os aprendizes estão prontos para trabalhar mais diretamente com desafios do conteúdo.

A presença do interesse influencia positivamente a atenção do aprendiz, o uso de estratégias e a definição de suas metas.

Interesse pode ser usado como apoio mesmo quando a pessoa inicialmente tem baixa autoeficácia, falta de metas e/ou não é capaz de se autorregular (op.cit., p.2).

\section{Autorregulação}

Refere-se ao processo pelo qual aprendizes focam seus pensamentos, sentimentos e ações para alcançar objetivos de aprendizagem.

Consiste em três processos: auto-observação, autojulgamento e autorreação.

1. Auto-observação significa dar atenção a seu próprio comportamento.

2. Autojulgamento significa comparar o nível atual de desempenho com as metas individuais.

3. Autorreação significa responder cognitivamente, afetivamente e comportamentalmente ao seu próprio autojulgamento (op.cit., p.116).

\section{Modelação Social e a AUtoeficácia de Bandura}

Na perspectiva da aprendizagem social de Bandura (2008), grande parte daquilo que o ser humano aprende se dá através da modelação social, na qual as pessoas padronizam seus estilos de pensamento e comportamento segundo exemplos de outras pessoas.

Essa modelação vem da aprendizagem por observação (observacional) ou por meio da aprendizagem por imitação. Mas os modelos construídos são dinâmicos, podem ser modificados e, inclusive, gerar novos modelos. Podem se estabilizar e funcionar como esquemas de assimilação ou serem abandonados se não funcionarem para o ser humano observador, modelador. 


\section{Determinismo Recíproco}

Na ótica da teoria social cognitiva, há um determinismo recíproco entre fatores pessoais (cognições, afetos e eventos biológicos), influências comportamentais (capacidades e habilidades) e fatores ambientais (contextuais).

Fatores ambientais podem ser muito importantes nesse determinismo, mas o ser humano não é simplesmente um produto de seu ambiente, de seu contexto social.

\section{AgÊnCIA HUMANA}

A teoria cognitiva social baseia-se em uma visão da agência humana, segundo a qual os indivíduos são agentes que podem fazer coisas acontecerem com seus atos e se envolvem de forma positiva em seu próprio desenvolvimento. Fundamental a esse sentido de agência há o fato de que, entre outros fatores pessoais, os indivíduos têm autocrenças que lhes permitem exercer um certo grau de controle sobre seus pensamentos, sentimentos e ações (Bandura et al., 2008, p.99)

Como agentes, as pessoas podem assumir mais controle de suas vidas por meio de mecanismos como a autorregulação e a autoeficácia.

\section{Autoeficácia}

Entre todos os pensamentos que afetam o funcionamento humano, localizados no núcleo fundamental da teoria social cognitiva, destacam-se as crenças de autoeficácia, julgamentos das pessoas sobre suas capacidades para organizar e executar cursos de ação necessários para alcançar determinados tipos de desempenho. Essencialmente, as crenças de autoeficácia são percepções que os indivíduos têm sobre suas próprias capacidades. Essas crenças de competência pessoal proporcionam a base para a motivação humana, o bem estar e as realizações pessoais. (Bandura et al., 2008, p.101)

Autoeficácia tem a ver com autoestima e capacidade de lidar com problemas da vida. Autoeficácia significa a crença que um indivíduo tem sobre sua capacidade de realizar com sucesso uma determinada atividade.

O fato de a pessoa acreditar que é capaz pode afetar tanto suas escolhas como seu desempenho, ou seja, determina como ela se sente, pensa, se motiva e se comporta. (Nascimento, 2016, p.33).

\section{Fontes De AUtoeficÁCiA}

São quatro as principais fontes de crenças de autoeficácia: experiência de domínio, experiência vicária, persuasões sociais e estados físicos e emocionais. 
1. Experiência de domínio é a interpretação do resultado do comportamento anterior do indivíduo; é o mais influente.

2. Experiência vicária é o que ocorre quando as pessoas formam crenças de autoeficácia observando outras pessoas executando tarefas e atividades.

3. Persuasões sociais são aquelas que as pessoas sofrem de outras pessoas, podendo ser positivas e cultivar crenças de autoeficácia ou negativas e enfraquecer essas crenças.

4. Estados físicos e emocionais como ansiedade, estresse, excitação, fadiga, dores, estados de humor também são fontes de autoeficácia, no sentido de que influenciam crenças de autoeficácia.

\section{Autoeficácia E ENSINO}

Crenças de autoeficácia têm grande influência no comportamento humano, naquilo que as pessoas fazem ou não fazem, desistem.

A autoeficácia tem um papel fundamental no processo ensino-aprendizagem. Por exemplo, estudantes com baixa autoeficácia podem acabar abandonando a escola. Na universidade podem optar por carreiras que não impliquem certas disciplinas que, em seu julgamento, não dariam conta delas, não seriam aprovados.

\section{A teOria dA CARGA COGNITIVA DE SWELler}

A teoria da carga cognitiva tem como conceitos/construtos centrais carga cognitiva, memória de trabalho e memória de longo prazo.

Não são novos, mas na perspectiva do ensino e da aprendizagem são muito relevantes.

Como ensinar sem inferir, minimamente, o que está passando na memória de trabalho de quem aprende? Como facilitar esse processo? Como chegam, ou não chegam, novos conhecimentos à memória de longo prazo?

\section{Memória de trabalho}

É a parte do cérebro humano que processa o que se está fazendo e pensando. É onde começa a aprendizagem de novos conhecimentos, declarativos ou procedimentais. Uma vez processados cognitivamente na memória de trabalho, esses conhecimentos podem ser transferidos à memória de longo prazo e lá "armazenados".

Mas esses novos conhecimentos podem ser descartados, ou seja, não "guardados"na memória de longo prazo, porque a aprendizagem significativa sempre depende da intencionalidade de quem aprende.

\section{LimitAÇÃo DA MEMÓRIA DE TRABALHO (O MÁGICO NÚMERO SETE)}

George Miller (1920-2012), psicólogo cognitivo, estudando processos mentais, chegou ao resultado de que a memória de trabalho está limitada a processar, simultaneamente, 
somente 7 mais ou menos 2 bits, chunks, de informação. Este resultado cunhado por Miller (1956) como "o mágico número sete "segue vigente.

Essa limitação tem tudo a ver com a carga cognitiva do que está sendo processado ou se pretende processar.

Outro resultado importante em relação à memória de trabalho são os modelos mentais de Johnson-Laird (1983).

\section{Modelos Mentais}

Para dar conta de uma situação nova, um problema novo, a primeira ação cognitiva de um ser humano é construir um modelo mental na memória de trabalho.

Modelo mental é um análogo estrutural da situação-problema.

Modelo mental é recursivo, ou seja, pode ser modificado até que se torne funcional para quem está mentalizando. Pode ser descartado ou pode se estabilizar e ser levado para a memória de longo prazo e armazenado como um esquema de assimilação.

\section{MeMóRIA DE LONGO PRAZO}

Contrariamente à memória do trabalho, que é bastante limitada, a memória de longo prazo tem alta capacidade de armazenamento de conhecimentos; chega a ser considerada incomensurável.

Mas interpretar a memória de longo prazo como um repositório de conhecimentos, como uma parte do cérebro humano onde são "armazenados", "estocados", "guardados", conhecimentos adquiridos é uma concepção errônea dessa memória.

A memória de longo prazo é a estrutura central da cognição humana: é dinâmica, os conhecimentos que lá estão podem ser modificados, melhorados, obliterados; pode contar com muitos ou poucos conhecimentos dependendo das aprendizagens ocorridas.

\section{CARga COGNITIVA}

A carga cognitiva refere-se ao esforço cognitivo associado à aprendizagem de um determinado tópico, à resolução de uma tarefa, à construção de um modelo mental.

A carga cognitiva é dita intrínseca quando refere-se ao esforço associado à aprendizagem de um determinado tópico. É dita estranha (extrínseca) quando refere-se à maneira como a nova informação ou tarefa é apresentada ao aprendiz. É chamada germane quando está relacionada ao trabalho individual necessário para armazenar mentalmente um novo conhecimento.

As categorias intrínseca e estranha definem a carga cognitiva total de uma tarefa de aprendizagem. Reside aí um grande problema instrucional: se a carga cognitiva de uma tarefa for maior do que a capacidade de processamento da memória de trabalho, a aprendizagem é prejudicada ou, até mesmo, impedida.

Algumas tarefas de aprendizagem são intrinsecamente difíceis de serem aprendidas, particularmente porque implicam interatividade de vários elementos que devem ser proces- 
sados simultaneamente na memória de trabalho, a qual é limitada ao "mágico número sete ".

A categoria extrínseca também depende da interatividade de elementos na memória de trabalho, mas nesse caso os elementos não são implícitos às tarefas de aprendizagem, mas dependem da maneira como são propostas, das atividades nas quais devem se envolver os aprendizes.

A categoria germane, por sua vez, refere-se à própria memória de trabalho de quem aprende. São fontes, formas, de processamento de informação dos aprendizes.

\section{O ENSINO}

No ensino, na preparação dos materiais instrucionais, é indispensável levar em conta a carga intrínseca dos conteúdos (declarativos ou procedimentais) a serem trabalhados e a maneira (carga cognitiva estranha; extrínseca) como isso será feito a fim de reduzir a carga cognitiva total.

\section{Conhecimentos PRÉvios}

A memória de trabalho tem um papel fundamental na aprendizagem de novos conhecimentos. Diz-se que tudo começa na memória de trabalho. Mas como? Se a memória de trabalho é limitada, como se pode dizer isso?

Essa postura é metafórica, na prática a aprendizagem de novos conhecimentos depende da memória de longo prazo.

Para aprender, dar significado, entender o que está chegando à memória de trabalho, $\mathrm{o}$ aprendiz recorre, inevitavelmente, a conhecimentos que estão na memória de longo prazo, ou seja, a conhecimentos prévios.

De acordo com a arquitetura cognitiva humana, a base esquemática de conhecimentos existentes na memória de longo prazo representa o maior fator crítico influenciando a maneira como aprendemos novas informações. Estruturas de conhecimento previamente aprendidas constituem o fator que mais influencia a aprendizagem de novos materiais. (Kalyuga, 2011, pp. 47 e 59)

Segundo a Teoria da Carga Cognitiva, a magnitude da carga mental na aprendizagem depende de esquemas relevantes previamente construídos pelo aprendiz. (Plass et al., 2011)

David Ausubel (1963) dizia o mesmo desde a perspectiva da psicologia cognitiva: se fosse possível isolar uma variável como a que mais influencia a aprendizagem de novos conhecimentos, eu diria que é o conhecimento prévio do aprendiz. 


\section{ConClusão}

Seguramente o ensino de Física na educação contemporânea deve levar em conta a FMC (Física Moderna e Contemporânea), as TDICs (Tecnologias Digitais de Informação e Comunicação), a interdisciplinaridade (STEAM/Science, Technology, Engineering , Arts, and Mathematics), bem como questões de diversidade, equidade e gênero.

São grandes desafios, mas esforços nesse sentido não terão efeitos desejados se o ensino não despertar o interesse dos alunos pela Física, não aumentar a autoeficácia dos estudantes em relação ao domínio dos conteúdos físicos e não levar em consideração a carga cognitiva envolvida nas tarefas e problemas de Física.

A modo de conclusão, reitera-se que a proposta deste texto foi a de chamar atenção de professores (as) de Física, na Educação Básica e Superior, sobre a relevância dessas variáveis no ensino da Física voltado para aprendizagem significativa. Não foram sugeridas estratégias sobre como lidar com essas variáveis. Isso pode ser feito em outra oportunidade, mas o papel docente é fundamental, sejam quais forem as estratégias e recursos instrucionais disponíveis, professores (as) são essenciais para despertar e manter o interesse dos alunos, aumentar, e não baixar, sua autoeficácia e controlar a carga cognitiva extrínseca das tarefas de aprendizagem.

\section{REFERÊNCIAS}

Ausubel, D.P. (1963). The psychology of meaningful learning. New York, N.Y.: Grune and Stratton. $685 \mathrm{p}$.

Dewey, John (2015). Experience and Education. New York, N.Y.: Free Press. 91p. First edition 1938.

Dewey, John (2018). Democracy and Education. Gorham, ME: Myers Education Press. 386p. First edition 1916.

Renninger, K.A., Nieswandt, M., and Hidi, S. (Eds.) (2015). Interest in mathematics and science learning. Washington, D.C.: American Educational Research Association. 417p.

Bandura, A., Azzi, R.G, Polydoro, S. (2008). Teoria Social Cognitiva: conceitos básicos. Porto Alegre: Artmed. 176p.

Nascimento, L.C. (2016). Biografia Albert Bandura. Psicanálise. Grandes Temas do Conhecimento, n.23: 2635 .

Miller, G.A. (1956). The magical number seven, plus or minus two: some limits on our capacity for processing information. Psychological Review, 63(2):81-87.

Johnson-Laird, P. (1983). Mental models. Cambridge, MA: Harvard University Press. 513 p. 
Kalyuga, S. (2011). Schema acquisitions and sources of a cognitive load. In Sweller, J., Ayres P., and Kayluga, S. Cognitive Load Theory. New York, N.Y.: Springer. Pp. 48-64.

Sweller, J. (2011). Cognitive Load Theory, learning difficulty and instructional design. Learning and Instruction, 4:295-312.

Sweller, J. (1988). Cognitive load during problem solving: effects on learning. Cognitive Science, 12:257-285.

Plass, J.L., Moreno, R., and Brünken, R. (2010). Cognitive Load Theory. New York, N.Y.: Cambridge University Press. 275p. 